\title{
Morfología y vascularización del nervio radial del perro
}

\author{
Flores Quintana, C.I.; Llano, E.G.; Báez, A.D.; Cabrera, W.R.; Benítez Ruiz Díaz, J.S.
}

Cátedras de Histología y Embriología y Anatomía II, Facultad de Ciencias Veterinarias, Universidad Nacional del Nordeste, Sargento Cabral 2139, Corrientes (3400), Argentina, E-mail: anato2@vet.unne.edu.ar.

\begin{abstract}
Resumen
Flores Quintana, C.I.; Llano, E.G.; Báez, A.D.; Cabrera, W.R.; Benítez Ruiz Díaz, J.S.: Morfología y vascularización del nervio radial del perro. Rev. vet. 21: 2, 127-131, 2010. El objetivo del trabajo fue determinar el origen y distribución segmentaria de los vasos sanguíneos que constituyen el sistema extrínseco de vascularización e identificar el número y situación de los fascículos que conforman el nervio radial del perro. Se trabajó con diez miembros anteriores de caninos, que fueron inyectados con látex coloreado, procediéndose luego a disecar el nervio y su correspondiente irrigación. A partir de cortes transversales del tronco nervioso se realizaron preparados histológicos, determinándose por microscopía óptica el número y la distribución de los fascículos, agrupándose las fibras en tres categorías acorde a su diámetro (hasta 4, de 4 a 8 y de 8 a 12 um). Se efectuó también la medición del perímetro total del nervio y los diámetros correspondientes a cada fascículo que conformaban el tronco nervioso. La vascularización del nervio radial del perro en su segmento proximal, antes de contornear en espiral al húmero, está constituida por pequeños vasos que se originan de la arteria braquial profunda. En el segmento distal, antes de su división en ramas superficial y profunda, el nervio está irrigado por pequeños vasos que se originan de la arteria circunfleja humeral caudal. En cuanto a la conformación interna, se identificaron 2 fascículos en el segmento proximal y 4 en el distal; del análisis de sus fibras surgió que el $21 \%$ perteneció a la primera categoría, $34 \%$ a la segunda y $45 \%$ a la tercera. El perímetro total del nervio en su segmento proximal fue de 8.595 um y el diámetro de sus fascículos de 2.966 um (promedio). El perímetro total del nervio en su segmento distal fue de 8437 um y el diámetro de sus fascículos de 1.472 um (promedio). Los resultados obtenidos pueden ser útiles al momento de efectuar maniobras quirúrgicas y reparadoras en la región del brazo del perro.
\end{abstract}

Palabras clave: perro, nervio radial, irrigación, fascículos.

\begin{abstract}
Flores Quintana, C.I.; Llano, E.G.; Báez, A.D.; Cabrera, W.R.; Benítez Ruiz Díaz, J.S.: Morphology and vascularization of canine radial nerve. Rev. vet. 21: 2, 127-131, 2010. Purposes of this work were to identify the beginning and the segmentary distribution of the blood vessels which form the extrinsic system of vascularization of the radial nerve in dogs as well as to identify the number and situation of its fascicles. Ten thoracic limbs of dogs were injected with colored latex, and nerve and its irrigation were dissected. Cross sections of the nervous trunk were made, and slides were stained with hematoxylin and eosin, in order to define by means of optic microscopy the number and distribution of the fascicles. Fibers were grouped in three categories according to their diameter (up to 4, from 4 to 8 , and from 8 to $12 \mathrm{um}$ ). Total perimeter of the nerve and diameter of each fascicle were also determined. Irrigation of the radial nerve in the dog is supplied by small vessels that come from the deep brachial artery and humeral circumflex caudal artery. Regarding its internal conformation, two fascicles in the proximal segment and four fascicles in the distal segment were identified. From the analysis of the fibers it can be considered that $21 \%$ belongs to the first category, $34 \%$ to the second and $45 \%$ to the third. The total perimeter of the nerve in its proximal segment was $8,595 \mathrm{um}$, and diameter for both fascicles of 2,966 um (average). The total perimeter of the nerve in its distal segment was 8,437 um with an average diameter of the four fascicles of $1,472 \mathrm{um}$. The results of this work may be considered when surgical and reparative maneuvers will be performed in the forearm region of the dog.
\end{abstract}

Key words: dog, radial nerve, irrigation, fascicles. 


\section{INTRODUCCIÓN}

El sistema nervioso periférico está formado por nervios de diferente grosor y longitud que recogen los estímulos desde la periferia y transportan hacia ella la respuesta motora, secretora o vegetativa que se origine. La mayoría son mixtos, formados por haces paralelos de fibras nerviosas que pueden ser somáticas o viscerales (sistema nervioso autónomo) aferentes (sensitivas) o eferentes (motoras) ${ }^{15}$. Desde su origen hasta el receptor periférico, las fibras se organizan y agrupan en haces o fascículos que se unen y dividen en reiteradas ocasiones dando lugar a verdaderos plexos intraneurales. Los fascículos están unidos entre sí por tejido conectivo, espacios hísticos, canales linfáticos y nervi nervorum.

La organización de los axones dentro de un nervio no responde a una disposición paralela entre sí, los axones ocupan diferentes posiciones en los distintos fascículos a lo largo de su trayecto, consiguiendo una relación de vecindad con otros axones y una ubicación más periférica o más central de acuerdo al fascículo que se disponga a lo largo del recorrido. El número de fascículos y el área de cada uno de ellos aumenta o disminuye en las distintas regiones que transcurren, existiendo además, variaciones anatómicas tan frecuentes que son consideradas la regla y no la excepción ${ }^{3}$.

Los nervios periféricos tienen una rica vascularización, que podría ser superior a la del sistema nervioso central, con múltiples anastomosis que forman la red vascular intraneural, la cual está formada principalmente por arteriolas, capilares y vénulas. La disposición vascular está diseñada para un flujo ininterrumpido, sin importar la posición del nervio en relación con el tejido circundante. La vulnerabilidad de los axones del sistema nervioso periférico a los cambios vasculares es bien conocida ${ }^{11,23,25,30}$.

El bloqueo anestésico de nervios periféricos es una técnica muy difundida en la práctica clínica. Sus riesgos pueden ser atenuados con conocimiento anatómico profundo del nervio escogido, sus posibles variacio-

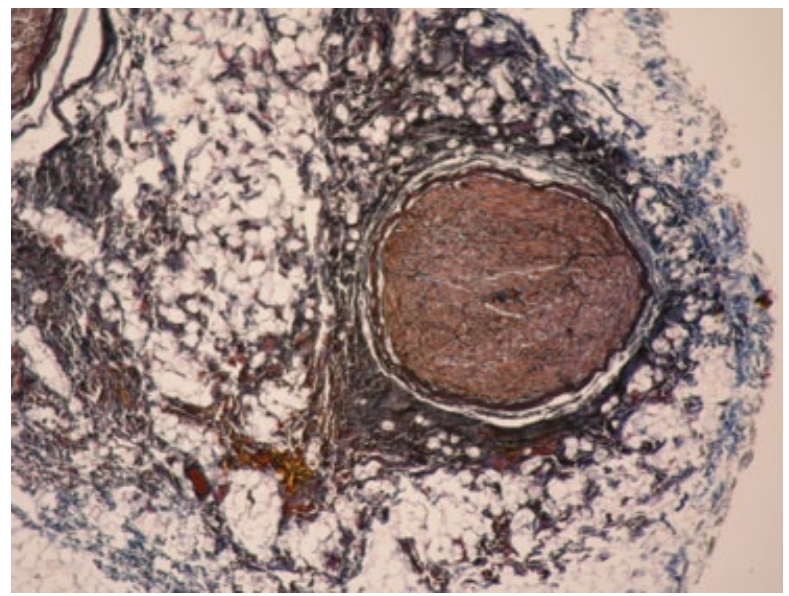

Figura 1. Nervio radial. Vista panorámica del epineuro. Mallory, 200x. nes morfológicas y relaciones con estructuras vecinas. Del mismo modo, la búsqueda de diferentes formas de abordaje y las nuevas técnicas y materiales disponibles ya sea para suturas o implantes destinados a la reparación y regeneración del nervio lesionado, necesitan de amplios conocimientos morfológicos. El análisis macro y microscópico de las estructuras que forman en su conjunto los nervios periféricos podría contribuir a un mejor entendimiento de los numerosos problemas observados clínicamente.

El objetivo del trabajo fue determinar el número y situación espacial de los fascículos que conforman el nervio radial del perro, permitiendo de esta manera su clasificación acorde a modelos de agrupación fascicular, e identificar el origen y distribución segmentaria de los vasos sanguíneos que constituyen el sistema extrínseco de vascularización.

\section{MATERIAL Y MÉTODOS}

Se utilizaron diez miembros anteriores de caninos, aplicándose la siguiente metodología: 1) sección del miembro separándolo del tórax; 2) localización y canalización de la arteria axilar; 3) inyección de pasta de repleción vascular (látex neoprene coloreado); 4) reposo de 48 h en cámara fría; 5) extirpación de toda la piel que cubría el miembro; 6) fijación del material por inmersión en formol al 10\%; 7) disección clásica del tronco nervioso, visualizando el origen de los vasos sanguíneos que constituyen el sistema extrínseco y su distribución segmentaria; 8) documentación fotográfica de las disecciones y esquematización de las observaciones registradas; 9) sección del tronco nervioso en una longitud de $1,5 \mathrm{~cm}$ colocando hilos de color rojo y azul que incluían el epineuro longitudinalmente en una extensión de $0,5 \mathrm{~cm}$, el hilo de color rojo indicó la posición lateral, el hilo de color azul la posición craneal; 10) fijación con formol buffer al $10 \%$ durante $24 \mathrm{~h}$; 11) inclusión en parafina; 12) realización de cortes con micrótomo tipo Minot a $5 \mathrm{um}$; 13) tinción con hematoxilina y eosina y tricrómica de Mallory, 14) observación

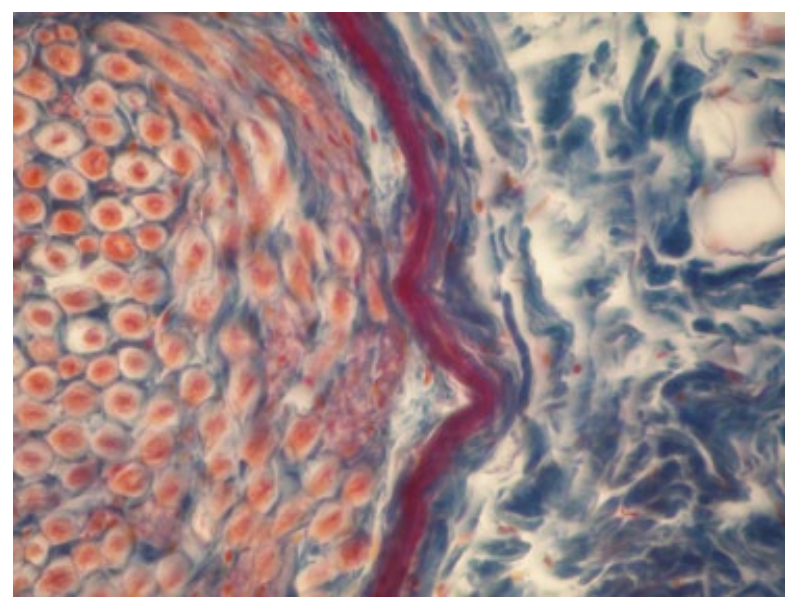

Figura 2. Nervio Radial. Fascículo del nervio mostrando parte del epineuro, perineuro y endoneuro. Mallory, 2000x. 
en microscopio óptico identificando el número y distribución fascicular 15) medición del perímetro total del nervio en cada segmento y del diámetro de los fascículos que lo conforman; 16) determinación del diámetro de 100 fibras en los principales fascículos y agrupación de las mismas en tres categorías, a partir de fotografías obtenidas diferenciadas en zonas periféricas y central de los fascículos, empleando el analizador de imagen Software Image Pro-Plus, versión 4.5, Media Cybernetics Inc.

\section{RESULTADOS Y DISCUSIÓN}

En la estructura del nervio se registró la presencia de tejido conectivo dispuesto en forma de vainas que organizaban la estructura interna del tronco nervioso. Estas vainas constituyeron el epineuro, tejido areolar que envuelve el nervio e incluye a los vasos intraneurales ${ }^{12,20,32}$, fácilmente identificable por tratarse de un nervio multifascicular ${ }^{1,29}$. Presentó fibras colágenas, las cuales pueden ser tipo I o tipos I y III ${ }^{16,26}$, dispuestas longitudinalmente respecto al eje de las fibras, fibras elásticas y tejido adiposo (Figura 1).

El perineuro, tejido denso multilamelar que delimita y envuelve cada fascículo, está formado por láminas de células perineurales concéntricas y fibras. El endoneuro, sustenta los fascículos y forma en éstos una delicada vaina que rodea las fibras mielínicas y amielínicas,

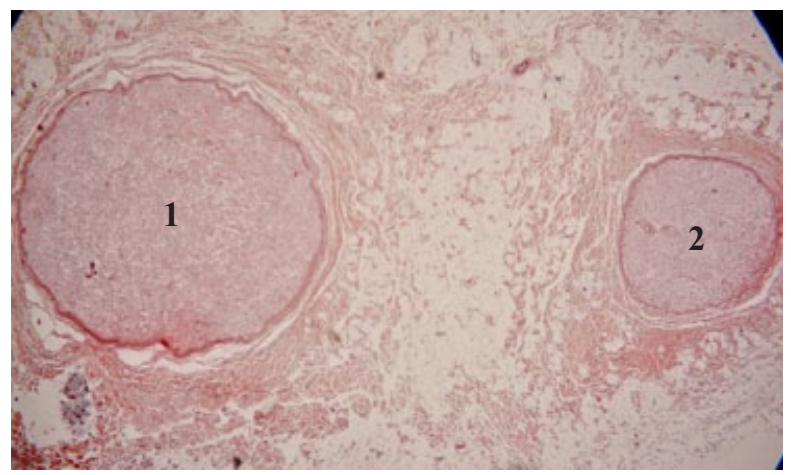

Figura 3. Nervio radial (segmento proximal). 1: fascículo craneal; 2: fascículo caudal. H y E, 500x.

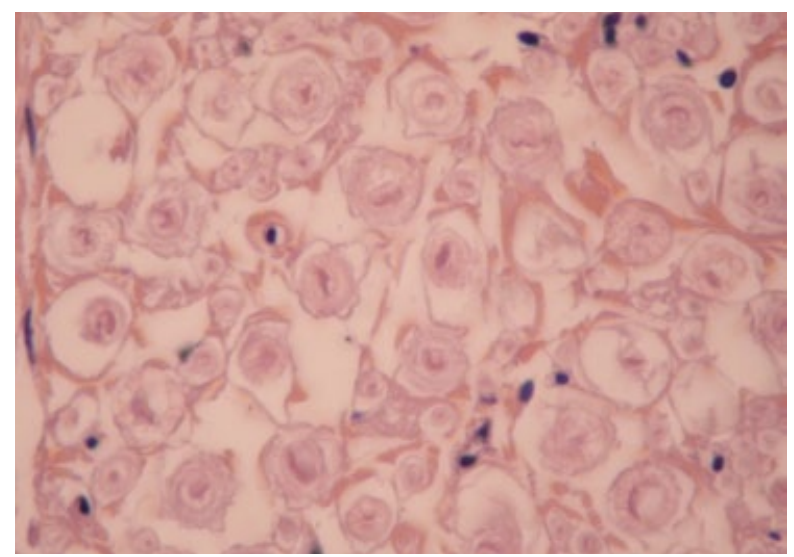

Figura 5. Fibras nerviosas en un fascículo del nervio radial segmento proximal. $\mathrm{H}$ y E, 5000x. pudiendo visualizarse con facilidad una de sus capas ${ }^{24}$ (Figura 2).

El perímetro promedio del segmento proximal fue 8.595 um, y se identificaron 2 fascículos: el fascículo craneal con diámetro de 3.852 um y el fascículo caudal de 2.081 um (Figura 3).

En el segmento distal el perímetro promedio fue 8.437 um y se identificaron 4 fascículos. El diámetro del fascículo craneal fue $1.100 \mathrm{um}$, el del fascículo intermedio fue $2.043 \mathrm{um}$, del fascículo caudal lateral fue 1.691 um y finalmente del fascículo caudal medial fue 1.053 um (Figura 4).

Tanto los fascículos como el epineuro son estructuras importantes al momento de realizar la sutura y aproximación de los segmentos dañados. Conocer la disposición fascicular en el uso de técnicas microquirúrgicas, ayudan a mejorar la especificidad de la reinervación y la recuperación funcional de los nervios $5,10,19,22$.

En el análisis de las fibras se registraron 3 categorías: categoría 1 (hasta 4 um de diámetro, donde se encontró el $40 \%$ de las fibras), categoría 2 (de 4 a 8 um donde se encontró el $46 \%$ de las fibras) y categoría 3 (más de 8 um donde se encontró el $14 \%$ de las fibras) (Figuras 5 y 6 ).

El aporte sanguíneo de un nervio periférico se origina en las arterias vecinas, vasos periósticos y musculares adyacentes más pequeños, que constituyen el

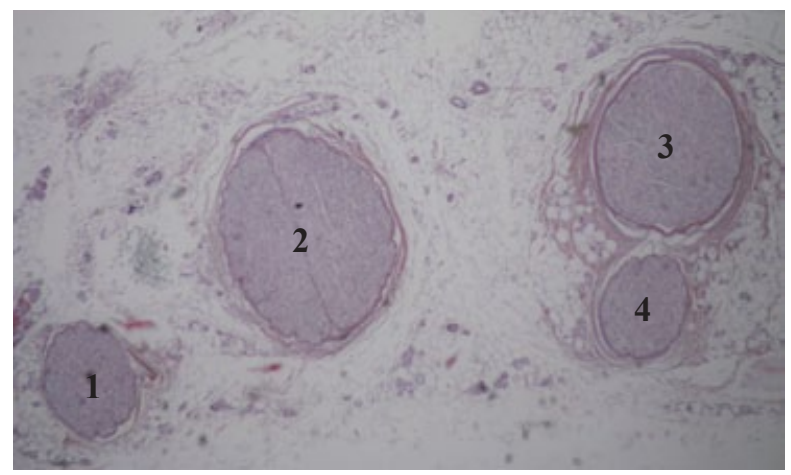

Figura 4. Nervio radial (segmento distal). 1: fascículo craneal; 2: fascículo intermedio; 3 : fascículo caudal lateral; 4: fascículo caudal medial. H y E, 500x.

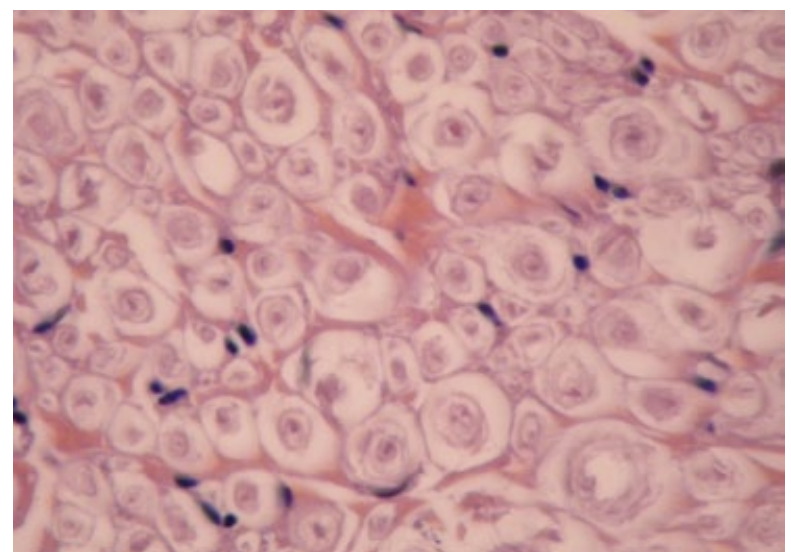

Figura 6. Fibras nerviosas en un fascículo del nervio radial segmento distal. H y E, 5000x. 
sistema extrínseco, y se dividen en ramas ascendentes y descendentes cuando alcanzan el epineuro. Estas mantienen una posición relativamente constante a lo largo de toda su longitud y aportan de forma mesoneural arterias nutricias que varían en tamaño y número, que penetran en el nervio a intervalos irregulares y se anastomosan con el sistema intrínseco 4, 5, 10, 19-22, 29, 31 . En el miembro torácico, el gran tronco arterial cambia su denominación a medida que atraviesa las diferentes regiones de la extremidad, la arteria subclavia se va transformando progresivamente en las arterias axilar, braquial y mediana ${ }^{18}$.

La arteria axilar se relaciona con los nervios del plexo braquial en la superficie medial de la articulación escápulo humeral y continúa distalmente como arteria braquial, que desciende medialmente por el brazo relacionándose en proximal con los nervios musculocu-

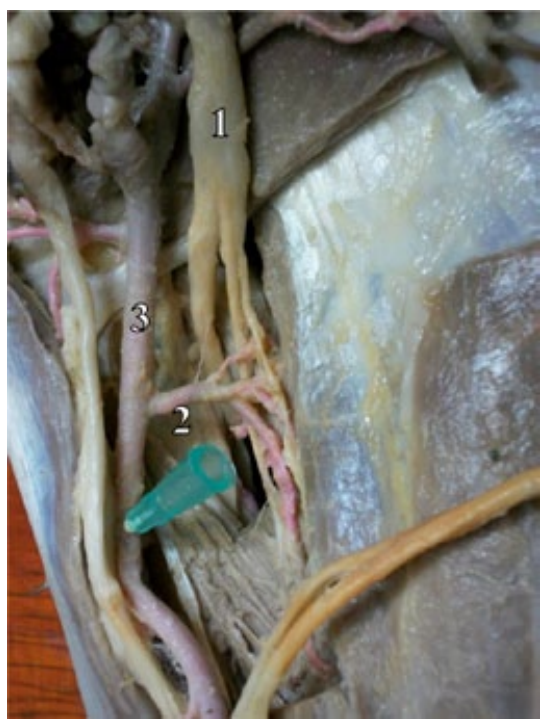

Figura 7. Irrigación del nervio radial (segmento proximal). 1: nervio radial; 2: arteria braquial profunda; 3 : arteria braquial.

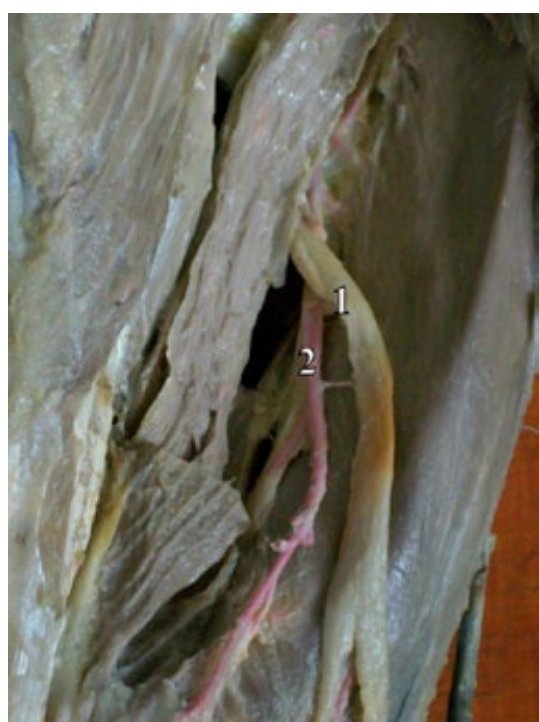

Figura 8. Irrigación del nervio radial (segmento distal). 1: nervio radial; 2: arteria humeral circunfleja caudal. táneo y radial, hacia distal se relaciona con el tronco combinado de los nervios mediano y cubital. La arteria braquial emite en su recorrido numerosas arterias colaterales entre ellas humeral circunfleja craneal, braquial profunda, bicipital, colateral ulnar, braquial superficial, transversa ulnar, recurrente ulnar e interósea común, continuándose finalmente como arteria mediana 2, 6-9, 13, 14, 17, 27, 28 .

El segmento proximal del nervio radial, antes de contornear en espiral al húmero está irrigado por pequeños vasos que se originan de la arteria braquial profunda (Figura 7).

El segmento distal del nervio radial antes de su división en troncos superficial y profundo está irrigado por pequeños vasos que se originan de la rama descendente de la arteria humeral circunfleja caudal (Figura 8).

Las descripciones efectuadas en este trabajo sobre la conformación fascicular e irrigación del nervio radial en el perro aportan al mejor conocimiento de la anatomía, brindando una contribución útil a la cirugía traumatológica, especialmente en las reparaciones nerviosas.

\section{REFERENCIAS}

1. Ayala Gutiérrez H, Colas C, Palacios JI, Morrondo JC. 2001. Cirugía secundaria de las lesiones de los nervios periféricos. On line: www.secpre.org/documentos.

2. Berg R. 1978. Anatomía topográfica y aplicada de los animales domésticos, Ed A.C., Madrid, p.354-378.

3. Bollini C. 2004. Revisión anatómica del plexo braquial. Rev Arg Anest 62: 386-398.

4. Cabrera WR, LLano EG, Baez AD. 2004. Irrigación de los nervios palmares y plantares en el equino. http://www. unne.edu.ar/web/cyt/com2004/index.htm.

5. Chrisman CH. 1996. Neuropatías periféricas. En: Fisiopatología y clínica quirúrgica en animales pequeños (Bojrab MJ Ed), Intermédica, Bogotá, p. 1192-1206.

6. Done SH, Godoy PC, Evans SA, Stickland NC. 1997. Atlas en color de anatomía veterinaria del perro y gato, Harcour Brace, Madrid, p. 4.2-4.47.

7. Dyce KM, Sack WO, Wensing CJ. 1999. Anatomía veterinaria, Panamericana, México, p. 30-32, 503-507.

8. Evans HE, Delahunta A. 2000. Disección del perro, McGraw-Hill, México, p.160-181.

9. Frandson RD. 1976. Anatomía y fisiología de los animales domésticos, Interamericana, México, p. 57-64.

10. Garibaldi L. 2003. Monoparesias y monoplejias. En: $E l$ libro de neurología para la práctica clínica (Pellegrino $\mathrm{F}$, Suraniti A, Garibaldi L, Ed), Intermédica, Buenos Aires, p. $161-167$.

11. Gelberman R, Szabo R, Williamson R, Dimick M. 1983. Sensibility testing in peripheral-nerve compression syndromes. An experimental study in humans. J Bone Joint Surg Am 65: 632-638.

12. Geneser F. 1990. Histología, Panamericana, Madrid, p. 296-298.

13. Getty R. 1982. Anatomía de los animales domésticos, Salvat, Barcelona, p. 209-221, 1863-1868. 
14. Gil J, Gimeno M, Laborda J, Nuviala J. 1997. Anatomía del perro, protocolos de disección, Masson, Madrid, p. $112-118$

15. Guyton AC. 1997. Anatomía y fisiología del sistema nervioso, Panamericana, Madrid, p. 123-125.

16. Junqueira L, Montes G, Kristzan R. 1979. The collagen of the vertebrate peripheral nervus system. Cell Tissue 202: 453-460.

17. Konig HE, Liebich HG. 2005. Anatomía de los animales domésticos, Panamericana, Buenos Aires, p. 253-261.

18. López Plana C, López Béjar M. 2004. Los miembros del perro: inervación y vascularización. On line: $h t t p: / / m i n n i e$. uab.es/ veteri/21202/atlas_virtual/primera.html.

19. Luhers Graca D, Riet-Correa G, Pioli Torres M. 2003. Citología del sistema nervioso periférico y su relevancia en las enfermedades neurológicas. En: El libro de neurología para la práctica clínica (Pellegrino F, Suraniti A, Garibaldi L, Ed), Intermédica, Buenos Aires, p. 622-626.

20. Marin Gutzke M, Castello Fortet JR, Santos H. 2001. Lesiones agudas de los nervios periféricos. On line: $w w w$. secpre.org/documentos.

21. Pellegrino F, Sánchez G. 1995. Anatomía funcional del sistema nervioso de los animales domésticos, Ed. AgroVet, Buenos Aires, p.13-37.

22. Pérez Fernández R, Labrador JM, Lara F, Marcos A, Hijano JC, Martínez R, Cabrera E. 2001. Injertos (cutáneos, dermograsos, tendinosos, nerviosos, óseos, cartilaginosos y vasculares). On line: www.secpre.org/documentos.
23. Powell H, Myers R. 1986. Pathology of experimental nerve compression. Lab Invest 55: 91-100.

24. Reina M, López A, Villanueva M, Andrés J, León G. 2000. Morfología de los nervios periféricos, de sus cubiertas y de su vascularización. Rev Esp Anestesiol Reanim 47: 464-475.

25. Rydevik B, Lundborg G. 1977. Permeability of intraneural microvessels and perineurium following acute, graded experimental nerve compression. Scand J Plast Reconstr Surg 11: 179-187.

26. Salonen V. 1987. Connective tissue reactions to peripheral nerve injury. Ann Univ Turk 28: 1-8.

27. Schaeller O. 1992. Nomenclatura anatómica veterinaria ilustrada, Acribia, Zaragoza, p. 322-325, 494-496.

28. Schwarze E. 1970. Compendio de anatomía veterinaria, Acribia, Zaragoza, p. 78-90.

29. Shores A. 2001. Sistema nervioso y órganos de la sensación. En: Técnicas actuales en cirugía de pequeños animales (Bojrab MJ, Ed), Intermédica, Montevideo, p. 67-76.

30. Sunderland S. 1976. The nerve lesion in carpal tunnel syndrome. J Neurol Neurosurg Psychiatr 39: 615-626.

31. Terraes AR, De Los Reyes MR, Gallovich JM, Recalde F, Romero E. 2001. Irrigación del plexus lumbalis. http:// www.unne.edu.ar/web/cyt/com2001/index.htm.

32. Ueyama T. 1978. The topography of root fibres within the sciatic nerve trunk of the dog. J Anat 127: 277-290. 